\title{
Primary Central Nervous System Lymphoma: A Real- World Comparison of Therapy Access and Outcomes by Hospital Setting
}

\author{
Akshat Maneesh Patel ( $\nabla$ akshat.patel@utsouthwestern.edu ) \\ The University of Texas Southwestern Medical Center https://orcid.org/0000-0002-8879-3046

\section{Omer Ali}

The University of Texas Southwestern Medical Center

\section{Radhika Kainthla}

The University of Texas Southwestern Medical Center

Syed M. Rizvi

The University of Texas Southwestern Medical Center

\section{Farrukh T. Awan}

The University of Texas Southwestern Medical Center

\section{Toral Patel}

The University of Texas Southwestern Medical Center

\section{Edward Pan}

The University of Texas Southwestern Medical Center

\section{Elizabeth Maher}

The University of Texas Southwestern Medical Center

Neil B. Desai

The University of Texas Southwestern Medical Center

Robert Timmerman

The University of Texas Southwestern Medical Center

Kiran A. Kumar

The University of Texas Southwestern Medical Center

\section{Praveen Ramakrishnan Geethakumari}

The University of Texas Southwestern Medical Center

\section{Research Article}

Keywords: primary CNS lymphoma (PCNSL), health services accessibility, healthcare disparities, practice patterns, outcomes

Posted Date: July 9th, 2021 
DOl: https://doi.org/10.21203/rs.3.rs-685267/v1

License: (c) (1) This work is licensed under a Creative Commons Attribution 4.0 International License. Read Full License

Version of Record: A version of this preprint was published at Neuro-Oncology Practice on January 12th, 2022. See the published version at https://doi.org/10.1093/nop/npab066. 


\section{Abstract}

Purpose:

Primary central nervous system lymphoma (PCNSL) is an aggressive disease with many tools for management that may be subject to resource barriers. This study compares the treatment patterns and survival outcomes among PCNSL patients treated at a safety-net hospital versus a tertiary academic institution.

\section{Methods:}

We retrospectively reviewed records of PCNSL patients from 2007-2020 $(n=95)$ at a public safety-net hospital $(n=33)$ and a private academic center $(n=62)$ staffed by the same university. Demographics, treatment patterns, and outcomes were analyzed.

\section{Results:}

Compared to the tertiary academic center, patients at the safety-net hospital were significantly younger, more commonly Black or Hispanic, and had a higher proportion of presenting patients with HIV. The safety-net hospital cohort was significantly less likely to receive induction chemotherapy ( $67 \% \mathrm{vs} 86 \%, p$ $=0.003)$ than those at the academic center. Safety-net hospital patients were significantly less likely to receive autologous stem cell transplant (ASCT) consolidation ( $0 \%$ vs. $44 \%, p=0.001)$ and had higher rates of consolidative WBRT ( $35 \%$ vs $15 \%, p=0.001$ ). Younger age and receiving consolidation were associated with improved progression-free survival (PFS, $p=0.001$ ) and overall survival (OS, $p=$ 0.001). Hospital location had no statistical effect on PFS $(p=0.725)$ or OS $(p=0.226)$ on age-adjusted analysis.

\section{Conclusions:}

Our study showed significant treatment differences between a public safety-net hospital and an academic cancer center reflecting access disparities. Despite variable treatment patterns, survival outcomes were not different. Further research is needed to determine optimal treatments for an orphan disease like PCNSL and it will be essential to advocate for equitable access in resource-limited settings.

\section{Introduction:}

Primary central nervous system lymphoma (PCNSL) is an aggressive, extranodal non-Hodgkin lymphoma, usually of diffuse large B-cell (DLBCL) histology, that is confined to the neuraxis and typically has a poor prognosis. PCNSLs are relatively rare tumors, accounting for $1-2 \%$ of all non-Hodgkin lymphomas [1]. PCNSL can occur in the setting of immunosuppression (patients living with HIV/AIDS [PLWH], organ transplant, immunosuppressive drugs), though the incidence has been rising among immunocompetent adults over 60 years of age [2]. 
The treatment of PCNSL typically consists of induction chemotherapy and subsequent consolidation therapies $[3,4]$. Despite general acceptance of induction regimens centered around high-dose methotrexate as cornerstones of treatment, consolidation strategies have not been optimized given the paucity of PCNSL [3-6]. Therefore, treatment practices across institutions vary regionally and globally. Additionally, optimal treatment of PCNSL may be subject to resource barriers; data show an increased rate of omitting chemotherapy in patients with markers of low socioeconomic status, specifically lack of insurance and median income [7]. Further, autologous stem cell transplantation (ASCT), a current consolidation option, is subject to cost barriers [8,9]. At safety-net hospitals, there are often access issues, delays in seeking medical care, and limited treatment options due to insurance barriers and other prohibitive costs.

This study compares the demographics, treatment patterns, and survival outcomes among PCNSL patients treated at a safety-net hospital versus a private academic hospital both treated by the same multidisciplinary team. We hypothesized that patients at our safety-net center would have different patterns of treatment compared to those at our tertiary academic institution.

\section{Methods:}

\section{Patients:}

(Hospital A) is an academic tertiary care center and National Cancer Institute ( $\mathrm{NCl}$ )-designated comprehensive cancer center, whose patient population is mainly privately insured and Medicare patients. (Hospital B) serves as a safety-net hospital mainly for uninsured residents of $* \star \star$. A multidisciplinary team of neuroradiologists, pathologists, neurosurgeons, neuro-, medical-, and radiation oncologists, serve at both clinical locations.

Medical records of patients treated for PCNSL from 2007-2020, at either a public safety-net hospital or an academic tertiary-care center, both serving the same metroplex, were analyzed via retrospective electronic chart review. Patients were excluded if they were diagnosed with systemic DLBCL with secondary CNS involvement. Patient demographics and disease characteristics were collected at the time of diagnosis. These include patient age, sex, race, Memorial Sloan-Kettering Cancer Center (MSKCC) prognostic class, and International Extranodal Study Group (IELSG) score. We additionally collected data on the induction and consolidation treatment modalities used.

\section{Treatment Characteristics:}

High dose methotrexate based (HD MTX) protocols were utilized in treating PCNSL at both institutions. HD MTX was typically given at 1-8 grams/m2/cycle depending on the specific protocol, patient's baseline renal and hepatic function, and patient tolerance. Standard hydration and urinary alkalinization with target $\mathrm{pH}$ of 7.5 was utilized in all HD MTX cases. Folinic acid rescue was provided 24 hours after each methotrexate dose and continued until serum methotrexate level was $\leq 0.10 \mathrm{uM}$. Following a 
positive response to induction HD MTX, patients could be offered consolidation autologous stem cell transplantation (ASCT), whole brain radiation therapy (WBRT), or further chemotherapy.

\section{Outcomes:}

The primary outcomes analyzed were overall (OS) and progression-free survival (PFS). OS was defined as the time from initial treatment to death resulting from any cause or last follow-up. PFS was defined as the time from initial treatment to MRI-confirmed disease progression, relapse, death, or last follow-up. Response was assessed by brain magnetic resonance imaging (MRI) per the International Primary CNS Lymphoma Collaborative Group (IPCG) criteria [10]. Patients were retrospectively evaluated for the incidence of neurological toxicity as documented by the treating provider.

\section{Statistical Analysis:}

Categorical variables and response rates were compared using Fisher's exact test. Continuous variables were compared using Mann-Whitney $\mathrm{U}$ test. Survival functions for overall- and progression-free survival (OS and PFS) were estimated by the Kaplan-Meier method and compared using a log-rank test. Cox proportional hazards regression was used for multivariable analysis to assess for confounding variables.

\section{Results:}

Patient and disease characteristics:

Median follow-up was 39 months for surviving patients and 11 months for all patients. Baseline characteristics of the study population are shown in Table 1. Compared to the tertiary academic center, patients at the safety-net hospital were significantly younger, had better MSKCC prognostic class, more commonly Black or Hispanic, and had a higher proportion of PLWH. 
Table 1

Baseline characteristics of patients at diagnosis

\begin{tabular}{|c|c|c|c|c|c|c|c|c|}
\hline \multirow{2}{*}{\multicolumn{2}{|c|}{ Characteristic }} & \multirow{2}{*}{\multicolumn{2}{|c|}{$\begin{array}{l}\text { Public Safety-Net } \\
\text { Hospital } \\
(n=33) \\
\text { N, \% or Median, IQR }\end{array}$}} & \multicolumn{2}{|c|}{$\begin{array}{l}\text { Private Academic } \\
\text { Center }(n=62)\end{array}$} & \multicolumn{3}{|c|}{$\begin{array}{l}\text { Total } \\
(n=95)\end{array}$} \\
\hline & & & & \multicolumn{2}{|c|}{$\mathrm{N}, \%$ or Median, IQR } & \multicolumn{2}{|c|}{$\begin{array}{l}\mathrm{N}, \% \text { or } \\
\text { Median, IQR }\end{array}$} & \multirow{2}{*}{$\begin{array}{l}\begin{array}{l}p- \\
\text { value }\end{array} \\
0.002\end{array}$} \\
\hline Age & & 48 & $38-59$ & 61 & $47-68$ & 56 & $44-65$ & \\
\hline \multirow{3}{*}{$\begin{array}{l}\text { Age } \\
\text { Groups }\end{array}$} & $<60$ & 26 & $79 \%$ & 30 & $48 \%$ & 56 & $59 \%$ & \multirow[t]{3}{*}{0.008} \\
\hline & $60-70$ & 6 & $18 \%$ & 19 & $31 \%$ & 25 & $26 \%$ & \\
\hline & $70+$ & 1 & $3 \%$ & 13 & $21 \%$ & 14 & $15 \%$ & \\
\hline KPS & & 70 & $50-90$ & 70 & $60-90$ & 70 & $58-90$ & 0.394 \\
\hline \multirow[t]{4}{*}{ Race } & Asian & 4 & $12 \%$ & 5 & $8 \%$ & 9 & $10 \%$ & \multirow{4}{*}{$<.001$} \\
\hline & Black & 8 & $24 \%$ & 4 & $7 \%$ & 12 & $13 \%$ & \\
\hline & Caucasian & 4 & $12 \%$ & 41 & $66 \%$ & 45 & $47 \%$ & \\
\hline & Hispanic & 17 & $52 \%$ & 12 & $19 \%$ & 29 & $31 \%$ & \\
\hline \multirow[t]{2}{*}{ Gender } & Female & 10 & $30 \%$ & 33 & $53 \%$ & 43 & $45 \%$ & \multirow[t]{2}{*}{0.051} \\
\hline & Male & 23 & $70 \%$ & 29 & $47 \%$ & 52 & $55 \%$ & \\
\hline $\begin{array}{l}\text { HIV } \\
\text { Status }\end{array}$ & PLWH & 15 & $46 \%$ & 3 & $5 \%$ & 18 & $19 \%$ & $\begin{array}{l}< \\
0.001\end{array}$ \\
\hline \multirow{3}{*}{$\begin{array}{l}\text { MSKCC } \\
\text { PC }\end{array}$} & Class I & 18 & $55 \%$ & 18 & $31 \%$ & 36 & $40 \%$ & \multirow[t]{3}{*}{0.091} \\
\hline & Class II & 8 & $24 \%$ & 24 & $41 \%$ & 32 & $35 \%$ & \\
\hline & Class III & 7 & $21 \%$ & 16 & $28 \%$ & 23 & $25 \%$ & \\
\hline \multirow{5}{*}{$\begin{array}{l}\text { ECOG } \\
\text { PS }\end{array}$} & 0 & 4 & $12 \%$ & 7 & $12 \%$ & 11 & $12 \%$ & \multirow[t]{5}{*}{0.898} \\
\hline & 1 & 10 & $30 \%$ & 22 & $39 \%$ & 32 & $36 \%$ & \\
\hline & 2 & 10 & $30 \%$ & 17 & $30 \%$ & 27 & $30 \%$ & \\
\hline & 3 & 5 & $15 \%$ & 7 & $12 \%$ & 12 & $13 \%$ & \\
\hline & 4 & 4 & $12 \%$ & 4 & $7 \%$ & 8 & $9 \%$ & \\
\hline \multirow{3}{*}{$\begin{array}{l}\text { IESLG } \\
\text { PS }\end{array}$} & low & 7 & $21 \%$ & 18 & $33 \%$ & 25 & $28 \%$ & \multirow[t]{3}{*}{0.358} \\
\hline & intermediate & 22 & $67 \%$ & 28 & $51 \%$ & 50 & $57 \%$ & \\
\hline & high & 4 & $12 \%$ & 9 & $16 \%$ & 13 & $15 \%$ & \\
\hline
\end{tabular}




\begin{tabular}{|llcccccc|}
\hline & $\begin{array}{l}\text { Public Safety-Net } \\
\text { Hospital } \\
(\mathbf{n = 3 3 )}\end{array}$ & $\begin{array}{l}\text { Private Academic } \\
\text { Center }(\mathbf{n = 6 2 )}\end{array}$ & \multicolumn{2}{l|}{$\begin{array}{l}\text { Total } \\
(\mathbf{n = 9 5 )}\end{array}$} & & \\
\hline $\begin{array}{l}\text { Deep structure } \\
\text { involvement }\end{array}$ & 23 & $70 \%$ & 31 & $50 \%$ & 54 & $57 \%$ & 0.083 \\
\hline Elevated Serum LDH & 11 & $33 \%$ & 19 & $31 \%$ & 30 & $32 \%$ & 0.820 \\
\hline
\end{tabular}

KPS: Karnofsky performance status. PLWH: Patients living with HIV. MSKCC PC: Memorial Sloan Kettering Cancer Center Prognostic Class. ECOG PS: Eastern Cooperative Oncology Group Performance Status. IELSG PS: International Extranodal Lymphoma Study Group Prognostic Score. LDH: Lactate dehydrogenase.

Induction modalities and response:

Figure 1 depicts the course of treatment and responses for our cohort by hospital setting.

Induction regimens used were either HD MTX based systemic chemotherapy (ST, $n=75$, median age 57 years) or WBRT ( $n=16$, median age 46 years). Four patients received only steroids and/or palliative care due to advanced disease/poor performance status at presentation (all treated at tertiary academic center, median age 65.5 years). Chemotherapy regimens were all HD MTX-based. The median number of cycles administered was 4 (range 1-9). Safety-net patients were significantly less likely to receive induction chemotherapy $(22 / 33[66.7 \%]$ vs $53 / 62$ [85.5\%], $p=0.003)$. Of patients who received induction ST, overall response rate (PR or CR) was $68.9 \%$. There was no significant difference in response rate to chemo-based induction between safety-net and academic center patients $(77.3 \%$ vs $65.4 \%$, respectively, $p=0.414)$.

Within the total cohort, 28 (38\%) had complete response (CR) and 23 (31\%) had partial response (PR), while $23(31 \%)$ had either progressive disease (PD) or no response to therapy. There was no difference in distribution of PRs and CRs by hospital setting $(p=0.269)$.

Consolidation modalities:

Patients who had a positive response to induction chemotherapy $(n=51)$ received either consolidative WBRT $(n=11)$, ASCT $(n=15)$, non-myeloablative chemotherapy $(n=14)$, or no further treatment $(n=11)$ due to rapid disease relapse or poor performance status. When comparing safety-net and academic center patients, hospital location did not significantly affect whether patients received consolidation therapy following a response to induction $(82.4 \%$ vs. $76.5 \%$, respectively, $p=0.731)$. Patients over the age of 60 were significantly less likely to receive consolidation (9/16 [56.3\%]) when compared to younger patients $(31 / 35[88.6 \%], p=0.023)$.

Safety-net hospital patients were significantly less likely to receive ASCT ( $0 \%$ vs. $44.1 \%, p=0.001)$ and had higher rates of consolidative WBRT ( $35.3 \%$ vs $14.7 \%, p=0.001)$. 
Second-line and salvage treatments:

Fifteen patients received second-line treatment for relapsed or refractory disease (systemic therapy, $\mathrm{n}=9$ $[60 \%]$, a combination of systemic therapy and radiation $(n=3,[20 \%])$, or WBRT, $n=3[20 \%])$. Only patients at the academic center received combination regimes.

Survival:

The median PFS for our entire cohort was estimated to be 25 months. PFS rates at two years following diagnosis were $56 \%$ vs $43 \%$ at the safety-net and academic center hospitals, respectively, and were not significantly different ( $p=0.294$, Fig. 2A). Younger age significantly increased PFS ( $p=0.0017$, Fig. $2 B)$. When adjusted for the differences in age distributions in the two patient populations, hospital setting again was not associated with PFS ( $p=0.725$, Odds Ratio [OR]: $1.12,95 \%$ Confidence Interval [CI]: $0.60-$ 2.09).

Median OS was not reached in our overall cohort. There was no significant difference in OS between patients treated at the safety-net compared to the academic center on univariate comparison (2-year OS: $77 \%$ vs $55 \%, p=0.061$, Fig. $2 E$ ) or when adjusted for age ( $p=0.226$, OR: $1.66,95 \% \mathrm{Cl}: 0.73-3.77)$. Younger age was independently associated with higher OS ( $p=0.0012$, Fig. 2F).

When comparing by induction treatment strategy, neither PFS nor OS were significantly different between patients receiving HD MTX ST or upfront WBRT (2-year PFS: $56 \%$ vs $35 \%, p=0.28$, Fig. 2C; 2-year OS: $67 \%$ vs $55 \%, p=0.6$, Fig. $2 G$ ). Receiving any form of consolidation following a response to induction chemotherapy was significantly associated with improved PFS and OS ( $p<0.001$ for both). Comparing survival outcomes between consolidation strategies in patients who received them $(n=40)$ found no significant differences in PFS ( $p=0.843$, Fig. $2 \mathrm{D})$ or OS $(p=0.32$, Fig. $2 \mathrm{H})$ between WBRT (2-year PFS: 91\%, 2-year OS: 91\%), ASCT (2-year PFS: 72\%, 2-year OS: 81\%), or chemotherapy (2-year PFS: 85\%, 2-year OS: $100 \%)$.

PLWH Subset Analysis:

Our cohort included 18 confirmed PLWH, 15 of which were treated at the safety-net hospital. Of these, 11 received WBRT as initial treatment and 7 received upfront MTX-based chemotherapy. PLWH were less likely to receive chemotherapy $(38.9 \%$ vs $88.9 \%, p<0.001)$ and more likely to receive WBRT as initial treatment $(61.1 \%$ vs. $6.9 \%, p<0.001)$ when compared to the rest of the cohort. All 7 PLWH who received induction chemotherapy achieved an overall response (PR or CR). PLWH were overall younger (median age 40 vs $60, p<0.001)$ and had a higher proportion of MSKCC prognostic score $1(82.4 \%$ vs. $29.7 \%, p=$ 0.001 ).

Neurotoxicity: 
Eleven cases of neurotoxicity were documented in our cohort. All received initial MTX-based chemotherapy. Of these 11, 5 occurred in patients who did not have an initial response to induction chemo and did not receive consolidative therapy. Of the 6 instances that occurred in patients receiving consolidation, 3 were with WBRT, 2 with ASCT, and 1 with further chemo. Neurotoxicity rates did not differ between hospital settings $(15.2 \%$ vs $9.7 \%, p=0.506)$.

\section{Discussion:}

This study retrospectively compared PCNSL patients treated at two hospital settings by the same team of providers: a public safety-net hospital and a tertiary academic center, serving the same geographic region. We describe the differences in demographic characteristics and treatment patterns of patients at these institutions. Patients at the safety-net hospital were younger and trended towards a better baseline MSKCC prognostic class compared to those at the academic center. They also were more likely to be from a racial minority (Hispanic or Black) and be PLWH. The safety-net hospital patients less often received induction chemotherapy and did not receive a consolidative ASCT, with comparatively higher use of consolidative WBRT. Despite these treatment differences, there were no significant statistical differences in survival outcomes between the two cohorts. Further, both hospital settings did not significantly differ in the rates of neurotoxicity.

Treatment patterns differed in both induction and consolidation between the two hospital settings despite being treated by the same multidisciplinary team. However, the divergence in induction patterns seem to be tied to demographic differences of the populations treated. Our finding of overall lower usage of induction HD MTX in the safety-net setting in comparison to patients treated at the tertiary academic center can be strongly attributed to the high prevalence of PLWH (45.5\%) in our safety net cohort. HIV affects people with lower socioeconomic status (SES) at disproportionately high rates [11], which is reflected in our higher rates in the resource-limited population traditionally treated at the safety-net hospital. Patients' HIV status is an important factor in the treatment modality and survival of patients with PCNSL. In general, HIV associated lymphomas may present with more advanced disease and have decreased overall survival compared to HIV negative patients [2, 12]. Historically, HIV associated PCNSL patients have been shown to have received a lower percentage of chemotherapy [7]. This was reflected in our PLWH cohort, which were significantly less likely to receive induction HD MTX. This difference may be due to clinician decision to initially defer chemotherapy in PLWH who may have compromised immune status, concomitant opportunistic infections, and/or decreased functional reserve that resulted in pursuing non-chemotherapy treatment modalities such as WBRT. Research utilizing the National Cancer Database (NCDB) as well as single-center studies have demonstrated increases in chemotherapy application in PLWH over time, correlating with improved survival in this population, though studies hypothesize that this also corresponds to improving anti-retroviral therapy $[7,12,13]$. All PLWH in our cohort that received HD MTX had a PR or CR to induction (7/7). However, most PLWH (11/18) received WBRT as initial treatment, which led to our finding of higher upfront WBRT in our overall safety net cohort. 
Despite this treatment difference in induction therapy, OS and PFS were not statistically different in the safety net cohort when compared to the tertiary hospital cohort, which more frequently received HD MTX, and in fact trended towards better OS for safety net patients $(76.7 \%$ vs $54.7 \%, p=0.061)$ on univariate but not age-adjusted analysis. This is contrary to many reports which have definitively demonstrated better outcomes with chemotherapy in PCNSL over WBRT alone [14-20]. However, our patients receiving WBRT alone had a median age of 46 . We postulated that our safety-net cohort were skewed towards younger age due to our high proportion of PLWH (who were almost all under the age of 60 [17/18]), and therefore possibly tolerated radiation better than the historical comparisons, who were immunocompetent patients with median ages over 50 . To account for this confounder, we adjusted for variations in age, after which the difference in OS by hospital setting lost its trend towards significance. In resource-limited settings, our data suggest that younger PLWH can tolerate HD MTX (100\% response in our 7 patients), but also have good outcomes with conservative treatment with WBRT alone. Prospective trials should be conducted to optimize clinical decision-making for this population specifically, who are subject not only to a more advanced disease course, but also concomitant access issues due to deeply rooted ties between lower SES and HIV.

Our study also showed significant differences in patterns of consolidation treatment following a positive response to HD MTX based induction chemotherapy. Safety-net patients were more likely to receive WBRT or non-myeloablative chemotherapy as consolidation as they were unable to receive ASCT. In contrast with differences in induction, this disparity is most certainly due to access and cost issues, as only patients at the private academic center were able to receive this treatment. In a prior claims database analysis, the median 100-day total costs for ASCT were estimated at $\$ 99,899$ (interquartile range (IQR), $\$ 73,914-140,555)[9,21]$. Reports have described the barriers to access in stem cell transplantation, with age, sex, race, and insurance status all having been shown to affect likelihood of receiving transplantation $[8,21]$. With regards to insurance status, which we postulate as the primary reason our safety-net patients could not receive ASCT, the specific barriers could be related to delays in approvals or lack of coverage for this costly medical procedure.

Regardless of modality of consolidative therapy, receiving any form of consolidation therapy was a significant predictor of improved survival in our study. When comparing the effect of various modalities of consolidation on survival, prospective studies have shown similar efficacy between WBRT and ASCT $[6,22]$. Others have found second-line chemotherapy to be a significant predictor of prolonged survival [18]. Some reports favor ASCT over WBRT for consolidation, due to risks of radiation induced neurotoxicity, especially in patients older than 60 years old [10], while ASCT incurs more hematologic and associated severe infection risk. In our study, there was no statistical difference in the rate of neurotoxicity between the two hospital systems though this assessment was limited by its retrospective nature. Given that safety net patients were younger and had a higher percentage of receiving WBRT as a population, further studies in a larger population with standardized, validated assessment tools to assess the risk and severity of neurotoxicity are crucial to improve risk assessment and ensure optimal long-term outcomes for patients with PCNSL in resource-limited settings where WBRT may be the only economically feasible option. 
Despite these considerations, the optimal consolidation modality remains unknown, with institutional preferences often playing the deciding role. Our study found no differences in OS or PFS between our hospital settings or the various consolidation strategies employed in our patients after HD MTX induction. However, our negative findings could reflect differences in patient baseline characteristics due to a younger and fitter population at the safety-net hospital, where ASCT was not performed. Either way, consolidation of any type seems preferred in responders in good performance status over no consolidation, leaving flexibility according to resource availability.

Ultimately, future studies will be needed to optimize consolidation regimes in PCNSL. Beyond the current options (WBRT, ASCT, chemotherapy), novel targeted agents and immunotherapies are showing promise in relapsed/refractory PCNSL [23-25]. As these are investigated further, great care should be made to not only disseminate this knowledge beyond the academic community, but also ensure publicly funded hospitals are equipped to deliver consolidation modalities regardless of cost barriers that may exist (particularly in ASCT). Resource-limited settings usually cater to a vulnerable patient population and clinicians and hospital systems will greatly benefit if provided the information and means to provide the most efficacious therapies.

This study is limited by its retrospective design, the small sample size within each treatment group, and heterogeneity in induction chemotherapy protocols between the hospital systems. Hospital setting had significant collinearity with PLWH, younger age, and omission of chemotherapy in the safety net cohort, making it difficult to ascertain each component's true effect on our survival outcomes. Inconsistencies in documentation between different hospital settings could potentially have masked any subtle differences between our cohorts. Neurotoxicity data was not collected in a standardized fashion and was based on different treating provider documentation, potentially obfuscating the true rates of neurotoxicity and making comparisons between our cohorts difficult. However, we report a comparatively large cohort and accurately document overall trends in treatment patterns and outcomes.

\section{Conclusion:}

PCNSL is an extranodal lymphoma with varying risk factors, diverse clinical presentations, and a heterogeneous approach to management. Our study shows that there are significant treatment differences between a public safety-net hospital and an academic cancer center reflecting socioeconomic and healthcare access disparities despite being cared for by the same oncological providers. Though survival outcomes were not different despite varying treatment patterns, many questions remain regarding best practices. Further research will be critical to determine how different risk groups (PLWH, other immunocompromised conditions, socioeconomic backgrounds, age, etc.) may be optimally managed using HD MTX, WBRT, ASCT, alternative non-myeloablative therapies, and targeted therapies. In addition, there is a dire need to evaluate neurocognitive effects at completion of treatment and the effects it has on quality of life in a standardized fashion. Once optimal strategies are elucidated, it is essential to advocate for equitable access to treatment strategies in resource-limited settings. 


\section{Declarations}

Funding: No funding was received for conducting this study.

Conflicts of interest/Competing interests: The authors have no relevant financial or non-financial interests to disclose.

Availability of data and material (data transparency): Please contact corresponding author for full database.

Code availability (software application or custom code): Not applicable.

Authors' contributions: All authors contributed to the study conception and design. Material preparation, data collection and analysis were performed by AP and OI. The first draft of the manuscript was written by $A P$, and all authors commented on previous versions of the manuscript. All authors read and approved the final manuscript. This project was supervised by KK and PRG.

Ethics approval: This IRB-approved retrospective chart review study involving human participants was in accordance with the ethical standards of the institutional research committee and with the 1964 Helsinki Declaration.

Consent to participate: Waived for retrospective review. Not applicable.

Consent for publication: Waived for retrospective review. Not applicable.

\section{References}

1. Swerdlow SH et al (2016) The 2016 revision of the World Health Organization classification of lymphoid neoplasms. Blood 127(20):2375-2390

2. Sandler AS, Kaplan LD (1996) Diagnosis and management of systemic non-Hodgkin's lymphoma in HIV disease. Hematol Oncol Clin North Am 10(5):1111-1124

3. Ferreri AJM et al., Evolving Treatments for Primary Central Nervous System Lymphoma. American Society of Clinical Oncology Educational Book, 2019(39): p. 454-466

4. Houillier $\mathrm{C}$ et al (2020) Management and outcome of primary CNS lymphoma in the modern era. An LOC network study 94(10):e1027-e1039

5. Houillier C et al (2019) Radiotherapy or Autologous Stem-Cell Transplantation for Primary CNS Lymphoma in Patients 60 Years of Age and Younger: Results of the Intergroup ANOCEF-GOELAMS Randomized Phase II PRECIS Study. J Clin Oncol 37(10):823-833

6. Ferreri AJM et al (2017) Whole-brain radiotherapy or autologous stem-cell transplantation as consolidation strategies after high-dose methotrexate-based chemoimmunotherapy in patients with primary CNS lymphoma: results of the second randomisation of the International Extranodal Lymphoma Study Group-32 phase 2 trial. Lancet Haematol 4(11):e510-e523 
7. Fallah J, Qunaj L, Olszewski AJ (2016) Therapy and outcomes of primary central nervous system lymphoma in the United States: analysis of the National Cancer Database. Blood advances 1(2):112-121

8. Mitchell JM et al (1997) Access to bone marrow transplantation for leukemia and lymphoma: the role of sociodemographic factors. J Clin Oncol 15(7):2644-2651

9. Majhail NS et al (2010) Access to Hematopoietic Cell Transplantation in the United States. Biol Blood Marrow Transplant 16(8):1070-1075

10. Abrey LE et al., Report of an international workshop to standardize baseline evaluation and response criteria for primary CNS lymphoma. J Clin Oncol, 2005. 23(22): p. 5034-43

11. Perry MJ (1998) Gender, race and economic perspectives on the social epidemiology of HIV infection: implications for prevention. The Journal of Primary Prevention

12. Olszewski AJ, Fallah J, Castillo JJ (2016) Human immunodeficiency virus-associated lymphomas in the antiretroviral therapy era: Analysis of the National Cancer Data Base. Cancer 122(17):2689-2697

13. Bayat A, Jones JC, Naina HVK (2014) Changing trend of HIV-associated PCNSL over 15 years: A single-center experience. J Clin Oncol 32(15_suppl):2049-2049

14. Schultz C et al (1996) Preirradiation chemotherapy with cyclophosphamide, doxorubicin, vincristine, and dexamethasone for primary CNS lymphomas: initial report of radiation therapy oncology group protocol 88 - 06. J Clin Oncol 14(2):556-564

15. Reni $\mathrm{M}$ et al (1997) Therapeutic management of primary central nervous system lymphoma in immunocompetent patients: results of a critical review of the literature. Ann Oncol 8(3):227-234

16. Laack NN et al (2006) Whole-brain radiotherapy and high-dose methylprednisolone for elderly patients with primary central nervous system lymphoma: Results of North Central Cancer Treatment Group (NCCTG) 96-73-51. Int J Radiat Oncol Biol Phys 65(5):1429-1439

17. Morris PG et al (2013) Rituximab, Methotrexate, Procarbazine, and Vincristine Followed by Consolidation Reduced-Dose Whole-Brain Radiotherapy and Cytarabine in Newly Diagnosed Primary CNS Lymphoma: Final Results and Long-Term Outcome. J Clin Oncol 31(31):3971-3979

18. Rubenstein JL et al (2013) Intensive Chemotherapy and Immunotherapy in Patients With Newly Diagnosed Primary CNS Lymphoma: CALGB 50202 (Alliance 50202). J Clin Oncol 31(25):30613068

19. Ferreri AJM et al (2016) Chemoimmunotherapy with methotrexate, cytarabine, thiotepa, and rituximab (MATRix regimen) in patients with primary CNS lymphoma: results of the first randomisation of the International Extranodal Lymphoma Study Group-32 (IELSG32) phase 2 trial. The Lancet Haematology 3(5):e217-e227

20. Kasenda B et al (2016) The role of whole brain radiation in primary CNS lymphoma. Blood 128(1):32-36

21. Majhail NS et al (2013) Costs of autologous and allogeneic hematopoietic cell transplantation in the United States: a study using a large national private claims database. Bone Marrow Transplant 48(2):294-300 
22. Houillier C et al (2019) Radiotherapy or Autologous Stem-Cell Transplantation for Primary CNS Lymphoma in Patients 60 Years of Age and Younger: Results of the Intergroup ANOCEF-GOELAMS Randomized Phase II PRECIS Study. J Clin Oncol 37(10):823-833

23. Ghesquieres $\mathrm{H}$ et al (2019) Lenalidomide in combination with intravenous rituximab (REVRI) in relapsed/refractory primary CNS lymphoma or primary intraocular lymphoma: a multicenter prospective 'proof of concept' phase II study of the French Oculo-Cerebral lymphoma (LOC) Network and the Lymphoma Study Association (LYSA)t. Ann Oncol 30(4):621-628

24. Ferreri AJM et al (2020) Improving the antitumor activity of R-CHOP with NGR-hTNF in primary CNS lymphoma: final results of a phase 2 trial. Blood Adv 4(15):3648-3658

25. Narita $Y$ et al (2021) Phase I/II study of tirabrutinib, a second-generation Bruton's tyrosine kinase inhibitor, in relapsed/refractory primary central nervous system lymphoma. Neuro Oncol 23(1):122133

\section{Figures}

\section{Patterns of Treatment}

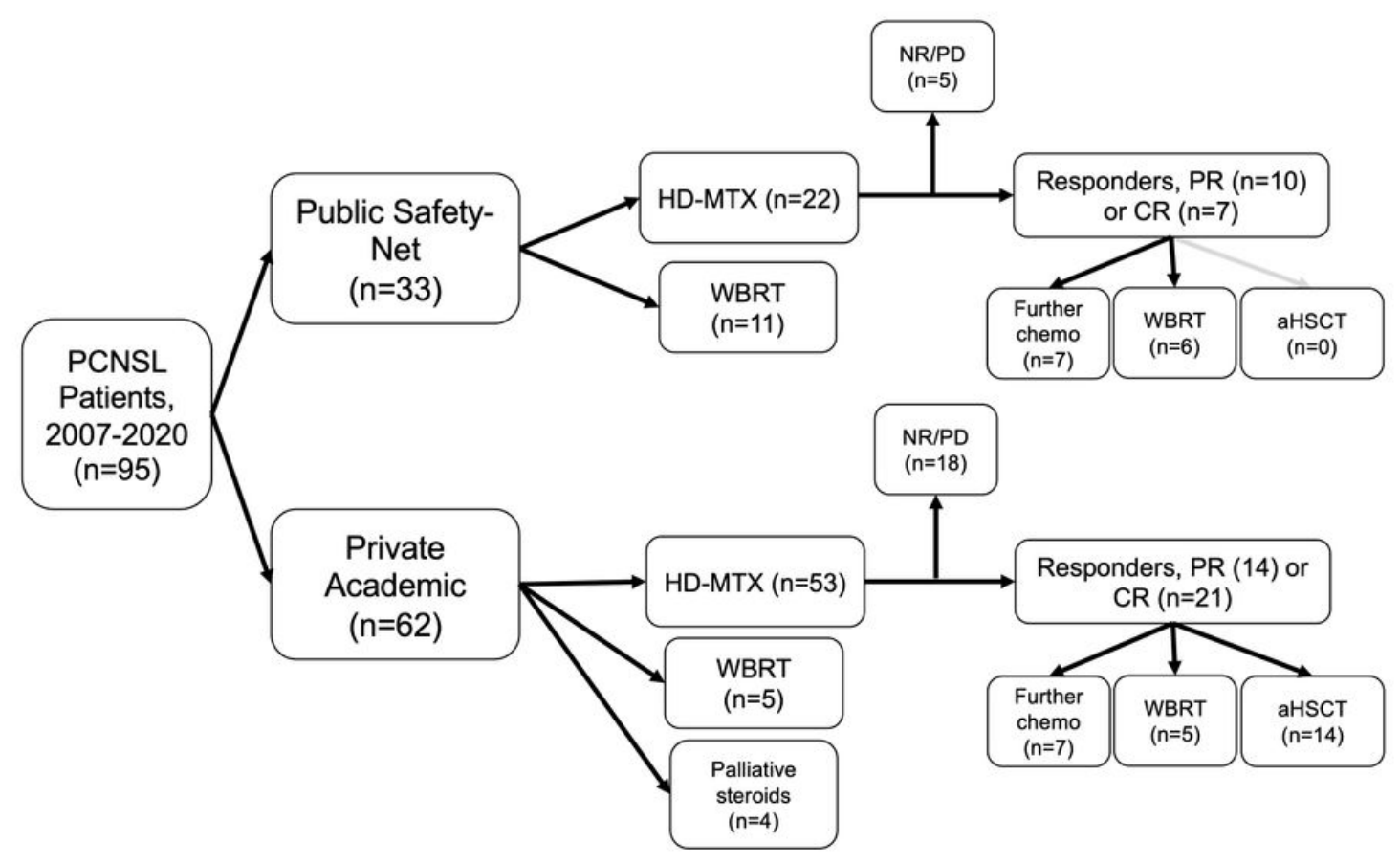

Hospital Setting Induction

Consolidation 
Figure 1

Treatment course of patients PCNSL: Primary CNS Lymphoma. HD-MTX: High-dose methotrexate. WBRT: Whole-Brian Radiation Therapy. NR/PD: No response/progressive disease. PR: Partial response. CR: Complete response. aHSCT: autologous hematopoietic stem cell transplant.

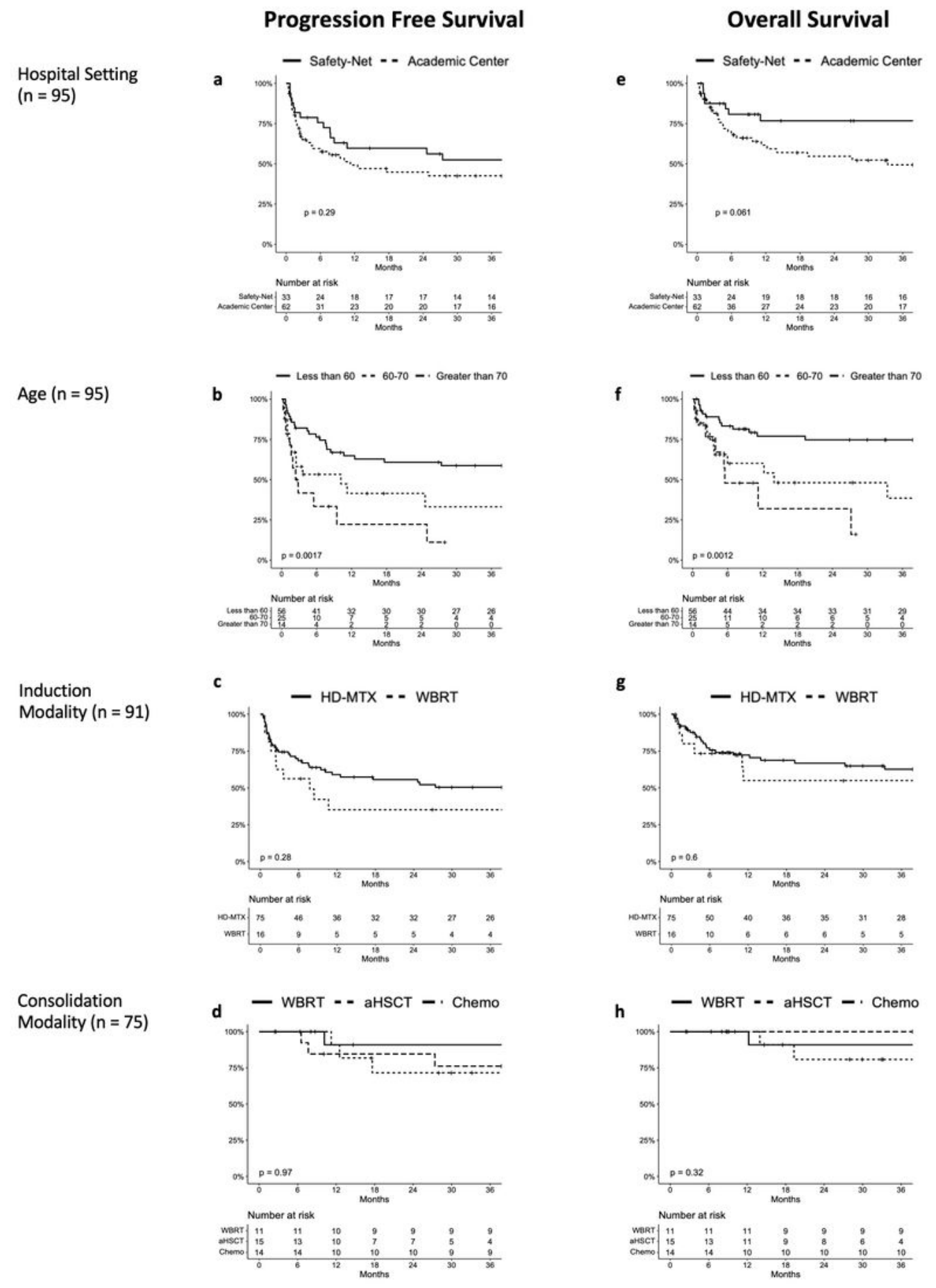

\section{Figure 2}


Survival curves by demographic and treatment factors PFS: Progression-free survival. OS: Overall survival. HD-MTX: High-dose methotrexate. WBRT: Whole-brain radiation therapy. aHSCT: autologous hematopoietic stem cell transplant. 